\title{
011 PREFERRED AND ACTUAL PLACE OF DEATH FOR PATIENTS REFERRED TO A SPECIALIST PALLIATIVE CARE SERVICE
}

Liz Arnold, Anne Finucane, Jenny Mullin, David Oxenham Marie Curie Hospice, Edinburgh, UK

10.1136/bmjspcare-2012-000196.11

Introduction The Department of Health's End of Life Strategy promotes high quality care for people approaching the end of life, including supporting patients to die in the place of their choice. Surveys suggest that the majority of the general population would choose to die at home. The authors present data on preferred place of death (PPD) for 1127 patients in contact with specialist palliative care services over a two year period. The authors examine whether preferences of hospice patients differ from those of the general population and whether documenting PPD relates to actual PPD. Methods: PPD data were collected prospectively as part of standard care for all patients referred to Marie Curie Hospice, Edinburgh. Case notes were reviewed retrospectively.

Results $77 \%$ of patients expressed a PPD. $60 \%$ chose hospice and $37 \%$ chose home; only $3 \%$ chose hospital or care home. $85 \%$ of patients died in their PPD. The proportion of patients who died in their PPD was higher for those wishing to die at the hospice $(93 \%)$ compared to those wishing to die at home (72\%). $17 \%$ of patients who did not express a preference died in hospital, compared to $6 \%$ of those with a documented place of death. 